\title{
Physical parameters and basis transformations in the two-Higgs-doublet model
}

\author{
C. C. Nishi \\ Instituto de Física “Gleb Wataghin” Universidade Estadual de Campinas, Unicamp 13083-970, Campinas, SP, Brasil \\ and Instituto de Física Teórica, UNESP-São Paulo State University Rua Pamplona, 145, 01405-900 - São Paulo, Brasil
}

(Received 27 December 2007; published 10 March 2008)

\begin{abstract}
A direct connection between physical parameters of general two-Higgs-doublet model (2HDM) potentials after electroweak symmetry breaking (EWSB) and the parameters that define the potentials before EWSB is established. These physical parameters, such as the mass matrix of the neutral Higgs bosons, have well-defined transformation properties under basis transformations transposed to the fields after EWSB. The relations are also explicitly written in a basis covariant form. Violation of these relations may indicate models beyond 2HDMs. In certain cases the whole potential can be defined in terms of the physical parameters. The distinction between basis transformations and reparametrizations is pointed out. Some physical implications are discussed.
\end{abstract}

DOI: 10.1103/PhysRevD.77.055009

PACS numbers: 12.60.Fr, 11.30.Qc, 14.80.Cp

\section{INTRODUCTION}

The standard model (SM) relies on the Higgs mechanism to give masses to all massive gauge bosons and elementary fermions. Such mechanism involves the spontaneous symmetry breaking (SSB) of the electroweak $S U(2)_{L} \otimes U(1)_{Y}$ gauge group to the electromagnetic $U(1)_{\mathrm{EM}}$ as a scalar Higgs doublet acquires a nonzero vacuum expectation value (VEV). Such scheme imposes universality constraints on the couplings between fermions and gauge bosons, establishes relations between the masses of the gauge bosons, and fixes the couplings between the physical Higgs and the fermions to be proportional to the masses of the latter [1].

The scalar potential, constituted by only one Higgs doublet, is also very restrictive since, from the knowledge of the electroweak (EW) VEV and the physical Higgs mass, the Higgs trilinear and quartic coupling constants are fixed at tree level. However, the physical Higgs boson, which is the only scalar remnant of the EW Higgs mechanism, has not yet been discovered. One then resorts to indirect means to bound the physical Higgs mass, most of them relying on the higher order perturbative behavior of the SM. Such bounds come from, e.g., the unitarity constraints of the scattering of gauge bosons, the validity of the SM up to the Planck scale, and the EW vacuum stability (see Ref. [1] and references therein). Studies constraining the Higgs mass are very important to its search, in particular, in view of the upcoming LHC experiment.

For models extending the electroweak symmetry breaking (EWSB) sector of the SM, the relation of the model parameters before EWSB and the physical parameters identified after EWSB may not be as minimal as in the SM. Specially for $N$-Higgs-doublet extensions of the SM (NHDMs), the multiplicity of independent parameters may be quite large due to the presence of a horizontal space, i.e., the space of identical gauge multiplets, in this case, $S U(2)_{L}$

*cnishi@ifi.unicamp.br doublets with quantum numbers identical to the SM Higgs doublet. The simplest two-Higgs-doublet model (2HDM) has been extensively studied recently [2-10] as the effective scalar sector of the MSSM requires two Higgs doublets for anomaly cancellation $[1,11]$. Historically, the addition of one or more Higgs doublets were considered to implement the spontaneous $C P$ violation mechanism (SCPV) $[12,13]$ as an alternative source of $C P$ violation.

Technical difficulties that arise when considering NHDMs are twofold: (i) more than one local minimum (orbit), not necessary with the same symmetry breaking pattern, might be present, even at tree level, and (ii) the reparametrization freedom [10] allowed by the presence of the horizontal space formed by the $N$-Higgs-doublets may masquerade the number of relevant independent parameters and symmetry properties such as $C P$ invariance. Difficulty (i) includes the possibility of potentials with no remaining $U(1)_{\mathrm{EM}}$ symmetry after EWSB (charge breaking vacuum) $[4,14,15]$ and it forces the stability of the vacuum to be a relevant issue at tree level [14].

Item (ii) concerns the reparametrization transformations induced by basis transformations (or horizontal transformations [16]) acting on the identical $N$-Higgs-doublets. Since all the doublets have the same gauge quantum numbers with respect to the SM gauge group, there is no change in the physical content of the theory if one rotates the fields in such space [17-19]. Such possibility may masquerade the number of relevant independent parameters in the theory. More crucially, transforming real parameters into complex parameters (for complex multiplets), $C P$ invariant theories can be disguised as $C P$ violating theories. This issue can be solved in an objective way by noting that $C P$ invariant theories remain $C P$ invariant through basis transformations but the corresponding $C P$ symmetry transformation also acts differently in different bases. As a practical way of distinguishing the $C P$ property of a theory, one can resort to the use of $C P$-odd basis invariants analogous to the Jarlskog invariant [20] in the quark sector of the $\mathrm{SM}$. In the context of NHDMs, many such invariants can 
be constructed [18] but sufficient conditions for $C P$ invariance using a minimum number of invariants could be formulated only for the potentials of 2HDMs [7,18,19,21,22] and 3HDMs [19].

Recent advances in the study of $2 \mathrm{HDM}$ include the result that at most two local minima can be present whenever there is a discrete set of minima in the orbit space $[8,23]$. Such result was obtained by using a Minkowski structure that emerges naturally in the space of the fields through suitable change of variables [9,19]. However, some controversy remains from numerical examples presenting more than two local minima [24]. Since the counting of the number of local minima can be a very difficult task, the upper bound of the number of minima is an important result. It was also proved for $2 \mathrm{HDMs}$ that (a) charge breaking vacua cannot coexist with a neutral vacuum $[4,9]$ and (b) spontaneously $C P$ violating vacua cannot coexist with $C P$ invariant vacuum $[4,8,9]$. The result (b) can be extended in a weaker version to NHDMs: a spontaneously $C P$ violating extremum always lies above a $C P$ invariant extremum if the latter exists [15]. The Minkowski structure can be also partially extended to general NHDM potentials [25].

Bearing these results in mind, the present article aims for two goals concerning the $2 \mathrm{HDM}$ potential: (i) to extract all the physical parameters identifiable only after EWSB and (ii) to study their properties under basis transformations. The first goal involves having a more direct connection between the parameters of the potential and the physical parameters after EWSB, confining ourselves to the case of neutral vacua. Since basis transformations are allowed before EWSB and they are usually involved to reach the physical basis, it would be desirable to have a basis covariant relation for the physical parameters, which leads to goal (ii). A systematic study of the physical parameters of 2HDMs, including the scalar self-interactions and the interactions of scalars with fermions and gauge bosons, was carried out in Ref. [6]. The basis covariance, however, was not extended to the fields after EWSB.

Another aspect of item (i) regards seeking a physical parametrization of the $2 \mathrm{HDM}$ potential by rewriting the parameters before EWSB in terms of the physical parameters. As explained in the end of Ref. [25], what prevents the utility of a parametrization depending on physical parameters, such as the masses of the physical charged and neutral Higgs bosons, is the possibility of the potential so defined possess another deeper minimum. The existence of at most two local minima already ameliorate the situation. Such parametrization also excludes by construction the potentials without nontrivial minima. For the cases we know there is only one local minimum and hence it is also the global one, such parametrization is unambiguous. The question that remains is to know if such parametrization can cover all possible $2 \mathrm{HDM}$ potentials containing only one global minimum.
The outline is as follows: in Sec. II we find the mass matrix for neutral scalars in the basis where the mass matrix for charged Higgs bosons is already diagonal. Relations between potential parameters and the mass matrices are found. In Sec. III, covariant relations between the mass matrices and the potential parameters are shown. In Sec. IV we show how to achieve the truly physical basis where all the mass matrices are diagonal, pointing out the distinction between basis transformations before and after EWSB. Finally, the results and physical implications are discussed in Sec. V. Some possibly useful material, including an alternative method to ensure a bounded below potential, is presented in the appendices.

\section{PHYSICAL PARAMETERS IN THE PCH BASIS}

A general 2HDM potential can be divided into its quadratic and quartic parts as

$$
V=V_{2}+V_{4}
$$

The quadratic part is usually written as

$$
V_{2}=Y_{a b} \Phi_{a}^{\dagger} \Phi_{b}, \quad a, b=1,2,
$$

where $Y$ is a Hermitian matrix and $\Phi_{a}=\left(\phi_{a 1}, \phi_{a 2}\right)$ are the Higgs doublets for which the notation $\phi_{a 1}=\phi_{a}^{(+)}$and $\phi_{a 2}=\phi_{a}^{(0)}$ is usually adopted when the vacuum preserves the electromagnetic symmetry. The quartic part can be conveniently written [19] as

$$
V_{4}=\frac{1}{2} \Lambda_{\mu \nu} r_{\mu} r_{\nu}, \quad \mu, \nu=0,1,2,3,
$$

where $\Lambda$ is a $4 \times 4$ real symmetric matrix while

$$
r_{\mu}=\frac{1}{2}\left(\sigma_{\mu}\right)_{a b} \Phi_{a}^{\dagger} \Phi_{b},
$$

for $\mu=0,1,2,3$, are real quadratic combinations of the doublets. The matrix $\sigma_{0} \equiv \mathbb{1}_{2}$ and $\sigma_{i}$ are the Pauli matrices. The quadratic variables $r_{\mu}=\left(r_{0}, \mathbf{r}\right)$ are functionally free except for the future light cone constraint $[9,25]$

$$
r_{0}^{2}-\mathbf{r}^{2} \geq 0, \quad r_{0} \geq 0 .
$$

The convention of summation over repeated indices is adopted with Euclidean metric. For example, $r_{\mu} r_{\mu}=r_{0}^{2}+$ $\mathbf{r}^{2}$. The Minkowski metric will not be used to avoid confusion and all indices will be written as lower indices, different than Refs. [9,25].

Using the variables $r_{\mu}$, the quadratic part of the potential in Eq. (2) can be cast into the form

$$
V_{2}=M_{\mu} r_{\mu},
$$

where $M_{\mu}$ has four independent components. The number of free parameters contained in $Y$ and $M$ are the same and they are indeed related by

$$
Y=M_{\mu} \frac{1}{2} \sigma_{\mu} \leftrightarrow M_{\mu}=\operatorname{Tr}\left[\sigma_{\mu} Y\right] .
$$


The most general unitary basis transformation group for the potential (1) is $S U(2)_{H}$. A general member of such group acts as

$$
\Phi_{a} \rightarrow \Phi_{a}^{\prime}=U_{a b} \Phi_{b}, \quad r_{i} \rightarrow r_{i}^{\prime}=R_{i j}(U) r_{j},
$$

with $R_{i j}=\frac{1}{2} \operatorname{Tr}\left[U^{\dagger} \sigma_{i} U \sigma_{j}\right]$, while $r_{0}^{\prime}=r_{0}$ is invariant. The basis transformation in Eq. (8), in turn, induces universal reparametrizations on the parameters such as $M_{i}$ $\left(\sim \Lambda_{0 i}\right)$ and $\Lambda_{i j}$ as

$$
M_{i}^{\prime}=M_{j} R_{j i}, \quad \Lambda_{i j}^{\prime}=\Lambda_{k l} R_{k i} R_{l j},
$$

in such a way that

$$
V\left(r^{\prime} ; M, \Lambda\right)=V\left(r ; M^{\prime}, \Lambda^{\prime}\right) .
$$

We want to parametrize the potential in terms of physical quantities that are defined after EWSB. Confining ourselves to neutral vacua, the first choice of physical parameters will be the masses of physical particles, i.e., one charged scalar and three neutral scalars. In addition, more parameters such as the EW vacuum expectation value (VEV), the mixing among the neutral scalars, and certain coupling constants will be necessary to completely parametrize the potential that requires 11 essential parameters.

To extract the physical masses, we need the quadratic part of the potential after EWSB that is induced by

$$
\Phi_{a} \rightarrow\left\langle\Phi_{a}\right\rangle+\Phi_{a},
$$

where $\left\langle\Phi_{a}\right\rangle$ is the vacuum expectation value (VEV) of $\Phi_{a}$, usually a c-number minimum of the potential in (1). The extremum equations are shown in appendix A. With the shift of Eq. (11), the quadratic part of the potential can be written as [25]

$$
\left.V_{2}\right|_{\mathrm{SSB}}=\Phi_{a}^{\dagger}\langle M\rangle_{a b} \Phi_{b}+\frac{1}{2} \Lambda_{\mu \nu} s_{\mu} s_{\nu},
$$

where

$$
s_{\mu}=\frac{1}{2}\left\langle\Phi_{a}\right\rangle^{\dagger}\left(\sigma_{\mu}\right)_{a b} \Phi_{b}+\text { H.c. },
$$

and $\langle\mathbb{M}\rangle$ is the mass squared matrix for the charged Higgs bosons, including the charged Goldstone. (The mass squared matrix will be denoted simply as "mass matrix" from this point on.) Such matrix can be calculated as [25]

$$
\langle\mathbb{M}\rangle=Y+\frac{1}{2} \sigma_{\mu} \Lambda_{\mu \nu}\left\langle r_{\nu}\right\rangle,
$$

where

$$
\left\langle r_{\mu}\right\rangle=\frac{1}{2}\left(\sigma_{\mu}\right)_{a b}\left\langle\Phi_{a}\right\rangle^{\dagger}\left\langle\Phi_{b}\right\rangle .
$$

In the physical charged Higgs (PCH) basis [25], for a neutral vacuum, the VEVs are simply

$$
\left\langle\Phi_{1}\right\rangle=\left(\begin{array}{l}
0 \\
0
\end{array}\right), \quad\left\langle\Phi_{2}\right\rangle=\left(\begin{array}{c}
0 \\
\frac{\mathrm{v}}{\sqrt{2}}
\end{array}\right),
$$

while the doublets after the shift (11) can be parametrized as

$$
\Phi_{1}=\left(\begin{array}{c}
h^{+} \\
\frac{1}{\sqrt{2}}\left(t_{1}-i t_{2}\right)
\end{array}\right), \quad \Phi_{2}=\left(\begin{array}{c}
G^{+} \\
\frac{1}{\sqrt{2}}\left(-t_{3}+i G^{0}\right)
\end{array}\right),
$$

where $v=246 \mathrm{GeV}$, is the electroweak VEV, $t_{i}, i=1,2$, 3 , are normalized neutral scalar fields, $h^{+}$is the physical charged Higgs and $G^{+}$and $G^{0}$ are the charged and neutral Goldstone fields, respectively. The Goldstone fields $G^{+}$ and $G^{0}$ are absorbed by the longitudinal $W^{+}$and $Z^{0}$ gauge bosons by the Higgs mechanism. For the VEV of Eq. (16), we have

$$
\left\langle r_{\mu}\right\rangle=\frac{v^{2}}{4}(1,0,0,-1) .
$$

In the PCH basis, the mass matrix for the charged scalars can be written

$$
\langle\mathbb{M}\rangle=\operatorname{diag}\left(m^{2}, 0\right),
$$

where the null eigenvalue corresponds to the charged Goldstone. We can divide the quadratic part of the potential of Eq. (12) into

$$
\left.V_{2}\right|_{\text {SSB }}=\left.V_{2}\right|_{\text {charged }}+\left.V_{2}\right|_{\text {neutral }} .
$$

For the charged fields we have

$$
\left.V_{2}\right|_{\text {charged }}=m^{2} h^{+} h^{-},
$$

while

$$
\begin{aligned}
\left.V_{2}\right|_{\text {neutral }}= & \frac{1}{2} m^{2}\left(t_{1}^{2}+t_{2}^{2}\right)+\frac{1}{2} \Lambda_{\mu \nu} s_{\mu} s_{\nu}, \\
& =\frac{1}{2} t_{i}\left(\mathcal{M}_{N}\right)_{i j} t_{j},
\end{aligned}
$$

where, using the parametrization of Eq. (17),

$$
s_{0}=-\frac{v}{2} t_{3}, \quad s_{i}=\frac{v}{2} t_{i} .
$$

The $3 \times 3$ matrix $\mathcal{M}_{N}$ is the mass matrix for the physical neutral scalars given by

$$
\begin{aligned}
\mathcal{M}_{N}= & m^{2} \operatorname{diag}(1,1,0)+\frac{v^{2}}{4} \tilde{\Lambda}+\frac{v^{2}}{4} \\
& \times\left(\begin{array}{ccc}
0 & 0 & -\Lambda_{01} \\
0 & 0 & -\Lambda_{02} \\
-\Lambda_{01} & -\Lambda_{02} & \Lambda_{00}-2 \Lambda_{03}
\end{array}\right),
\end{aligned}
$$

where $\tilde{\Lambda}=\left\{\Lambda_{i j}\right\}, i, j=1,2,3$. The physical neutral scalars will be orthogonal combinations of $t_{i}$, defined by the diagonalization of $\mathcal{M}_{N}$ in Eq. (25). The mass matrix $\mathcal{M}_{N}$ in the PCH basis can be also found in Eqs. (24) and (41) of Ref. [6] in a different notation.

From Eqs. (14), (18), and (25) we can find the following relation between $Y$ and $\mathcal{M}_{N}$,

$$
Y=\frac{1}{2}\left(\begin{array}{cc}
2 Y_{11} & \left(\mathcal{M}_{N}\right)_{13}-i\left(\mathcal{M}_{N}\right)_{23} \\
\left(\mathcal{M}_{N}\right)_{13}+i\left(\mathcal{M}_{N}\right)_{23} & -\left(\mathcal{M}_{N}\right)_{33}
\end{array}\right)
$$


Except for $Y_{11}$, all elements of $Y$ are directly related to $\mathcal{M}_{N}$.

Hence, we can completely parametrize the potential in the PCH basis in terms of the set of 12 parameters

$$
\left\{v, m, \Lambda_{00}, \mathcal{M}_{N}, \Lambda_{0}\right\},
$$

where $\Lambda_{\mathbf{0}}=\left\{\Lambda_{0 i}\right\}, i=1,2,3$. For fixed values for the set in Eq. (27), we obtain from Eq. (25) the rest of $\Lambda_{\mu \nu}$ by

$$
\begin{array}{r}
\Lambda_{i j}=\frac{4}{v^{2}}\left[\left(\mathcal{M}_{N}\right)_{i j}-m^{2} \delta_{i j}\right], \quad i, j=1,2, \\
\Lambda_{i 3}=\Lambda_{0 i}+\frac{4}{v^{2}}\left(\mathcal{M}_{N}\right)_{i 3}, \quad i=1,2 \\
\Lambda_{33}=-\Lambda_{00}+2 \Lambda_{03}+\frac{4}{v^{2}}\left(\mathcal{M}_{N}\right)_{33} .
\end{array}
$$

The quadratic parameter $Y_{11}$ depends on more parameters other than $\mathcal{M}_{N}$ as

$$
Y_{11}=m^{2}-\frac{v^{2}}{4}\left(\Lambda_{00}-\Lambda_{03}\right)+\frac{1}{2}\left(\mathcal{M}_{N}\right)_{33} .
$$

There are 12 free parameters. Among these, 11 are essential and can not be eliminated by reparametrization [19]. Nevertheless, one parameter can be removed by a remaining $U(1)$ reparametrization freedom due to

$$
\Phi_{1} \rightarrow e^{i \theta} \Phi_{1}, \quad \Phi_{2} \rightarrow \Phi_{2} .
$$

Since

$$
h^{+} \rightarrow e^{i \theta} h^{+}
$$

represents the electromagnetic $U(1)_{\mathrm{EM}}$ invariance of the potential, the transformation of Eq. (32) amounts effectively to a $S O(2)$ rotation in the $t_{1}, t_{2}$ fields. Notice that since $\left\langle\Phi_{1}\right\rangle=0$, the transformation of Eq. (32) does not affect the VEVs. Choosing appropriately $\theta$ in Eq. (32), one can set one of the following parameters to zero: $\left(\mathcal{M}_{N}\right)_{12},\left(\mathcal{M}_{N}\right)_{13},\left(\mathcal{M}_{N}\right)_{23}$. In particular, choosing $\left(\mathcal{M}_{N}\right)_{23}=0$, we obtain a real symmetric $Y$ and we constrain $\Lambda_{23}=\Lambda_{03}$. For any choice the overall number of free independent parameters should be 11. Of course, different choices, such as $\Lambda_{01}=0$ or $\Lambda_{02}=0$, could be alternatively chosen. Obviously, once a choice is made, one cannot set more than one parameter to zero.

To ensure the vacuum in Eq. (17) is a local minimum, it is sufficient to pick positive values for $m^{2}$ (the mass squared of $h^{+}$) and for the three eigenvalues of $\mathcal{M}_{N}$ (the masses squared of $t_{i}$ ). Such requirements guarantee that the second derivative of the potential around the extremum is positive semidefinite.

There remains the question of boundedness for the potential defined with physical parameters (27). Firstly, we have to choose $\Lambda_{00} \geq 0$ because taking $r_{0} \rightarrow \infty$ but $|\mathbf{r}|$ finite in Eq. (3) would make $V_{4}$ acquire negative values if $\Lambda_{00}<0$. Moreover, the following statement can be proved: For a potential $V(r)$ defined as Eq. (1), satisfying $\Lambda_{00}+\lambda_{i}>0$ for all $\lambda_{i}, i=1,2,3$, eigenvalues of $\tilde{\Lambda}$, it is always possible to obtain $\Lambda_{\mu \nu} r_{\mu} r_{\nu}>0$, for all $r_{\mu}$ satisfying Eq. (5), by making the substitution

$$
\Lambda_{0} \rightarrow c \Lambda_{0}, \quad c>0,
$$

with appropriately small $c$. The proof is shown in appendix B.

The only problem that could make such physical parametrization not viable is the possibility that the potential defined for a given set (27) possesses another minimum that lies deeper than the one defined in (16). This possibility is real and numerical examples can be quickly devised. The problem is not so severe because at most two distinct local minima are possible for bounded below potentials containing two Higgs doublets [8]. Although, in Ref. [24], some numerical examples of 2HDM potentials with more than two minima were apparently devised [24]. On the other hand, this parametrization excludes potentials without nontrivial minima by construction.

\section{PHYSICAL PARAMETERS IN AN ARBITRARY BASIS}

For a general potential (1), the vacuum expectation value (VEV) will not be in the form of Eq. (16). Nonetheless, we can always parametrize a neutral vacuum as

$$
\left\langle\Phi_{1}\right\rangle=\frac{v}{\sqrt{2}}\left(\begin{array}{c}
0 \\
\cos \frac{\theta_{\mathrm{v}}}{2}
\end{array}\right), \quad\left\langle\Phi_{2}\right\rangle=\frac{v}{\sqrt{2}}\left(\begin{array}{c}
0 \\
e^{i \xi} \sin \frac{\theta_{\mathrm{v}}}{2}
\end{array}\right) .
$$

The VEV in Eq. (18) corresponds to $\theta_{v}=\pi$ and $\xi=\pi$. In the MSSM, the angle $\theta_{v}$ corresponds to $2 \beta$.

To explicit the structure of the horizontal space where basis transformations act, it is more convenient to define [25]

$$
u \equiv\left(\phi_{11}, \phi_{21}\right)^{\top}, \quad w \equiv\left(\phi_{12}, \phi_{22}\right)^{\top} .
$$

We can rewrite Eq. (35) as

$$
\langle u\rangle=(0,0)^{\top}, \quad\langle w\rangle=\frac{v}{\sqrt{2}}\left(\cos \frac{\theta_{\mathrm{v}}}{2}, \sin \frac{\theta_{\mathrm{v}}}{2} e^{i \xi}\right)^{\top} .
$$

More generally, we can rewrite

$$
\langle w\rangle=\frac{v}{\sqrt{2}} U_{v} e_{2},
$$

where $U_{v}$ is a unitary matrix in $S U(2)_{H}$ and $e_{a}, a=1,2$, are the canonical vectors defined by $\left(e_{a}\right)_{b}=\delta_{a b}$. In terms of $r_{\mu}$ we get

$$
\left\langle r_{\mu}\right\rangle=\frac{v^{2}}{4}\left(1, \cos \xi \sin \theta_{v}, \sin \xi \sin \theta_{v}, \cos \theta_{v}\right)=\frac{v^{2}}{4} R_{\mu \nu} n_{\nu},
$$

where $n_{\mu}=(1,0,0,-1)$ and $R_{\mu \nu}$ can be related to $U_{v}$ by 


$$
R_{\mu \nu}\left(U_{v}\right) \equiv \frac{1}{2} \operatorname{Tr}\left[U_{v}^{\dagger} \sigma_{\mu} U_{v} \sigma_{\nu}\right]=\left(\begin{array}{cc}
1 & \mathbf{0} \\
\mathbf{0} & \tilde{R}
\end{array}\right),
$$

with $\tilde{R}=\left\{R_{i j}\right\}, i, j=1,2,3$ being a rotation matrix in $S O(3)_{H}$.

We can rewrite the quadratic part of the potential in Eq. (12) using $u$ and $w$ of Eq. (36), and their respective VEVs in Eq. (37),

$$
\left.V_{2}\right|_{\mathrm{SSB}}=u^{\dagger}\langle\mathbb{M}\rangle u+w^{\dagger}\langle\mathbb{M}\rangle w+\frac{1}{2} \Lambda_{\mu \nu} s_{\mu} s_{\nu},
$$

where

$$
s_{\mu}=\frac{1}{2}\langle w\rangle^{\dagger} \sigma_{\mu} w+\text { H.c. }
$$

The first term of Eq. (41) corresponds to the mass term of the charged scalars, one physical and one Goldstone, in an arbitrary basis. The respective mass matrix is $\langle\mathbb{M}\rangle$, which is defined by Eq. (14).

The relation between $\langle\mathbb{M}\rangle$ in an arbitrary basis and its diagonal form (19) in the PCH basis is given by

$$
U_{v}^{\dagger}\langle\mathbb{M}\rangle U_{v}=\operatorname{diag}\left(m^{2}, 0\right) .
$$

We can then reach the PCH basis by the substitutions

$$
\Phi_{a}=\left(U_{v}\right)_{a b} \Phi_{b}^{\prime}, \quad\left\langle\Phi_{a}\right\rangle=\left(U_{v}\right)_{a b}\left\langle\Phi_{b}^{\prime}\right\rangle,
$$

or equivalently

$$
w=U_{v} w^{\prime}, \quad u=U_{v} u^{\prime},
$$

with the same substitutions valid for their respective VEVs.

Since the basis for which $\{Y, \Lambda,\langle r\rangle\}$ is defined is completely arbitrary, the covariance of $\langle\mathbb{M}\rangle$ is valid between any basis and not only with respect to the PCH basis. (A detailed account of the basis covariance of $\langle\mathbb{M}\rangle$ is given in appendix D.) Indeed, we can write [25]

$$
\langle\mathbb{M}\rangle=m^{2}\left[\mathbb{1}_{2}-\langle\hat{w}\rangle\langle\hat{w}\rangle^{\dagger}\right],
$$

where $\langle\hat{w}\rangle=\langle w\rangle /|\langle w\rangle|$.

We can try to extend the basis covariance for the mass matrix for the physical neutral scalars. We keep the notation $\mathcal{M}_{N}$ to denote such mass matrix. Obviously, the second and third term of Eq. (41) is covariant by basis transformations for $w$, such as the transformation (45) with arbitrary $U_{v}$. The question, however, is if we can find appropriate fields $t_{i}$ with suitable transformation properties that render $\mathcal{M}_{N}$ covariant by some basis transformation, keeping Eq. (23) form invariant. We obviously want to recover Eq. (25) for $\mathcal{M}_{N}$ and Eq. (24) for $t_{i}$ in the PCH basis.

The immediate extension of Eq. (17) to define $t_{i}$ in any basis is flawed because a basis transformation over $w$ in Eq. (17) would mix $t_{i}$ with the neutral Goldstone $G^{0}$. In other words, with $w^{\prime}$ in the $\mathrm{PCH}$ basis given by

$$
w^{\prime}=\frac{1}{\sqrt{2}}\left(\begin{array}{c}
t_{1}^{\prime}-i t_{2}^{\prime} \\
-t_{3}^{\prime}+i G^{0}
\end{array}\right),
$$

we cannot define

$$
w=\frac{1}{\sqrt{2}}\left(\begin{array}{c}
t_{1}-i t_{2} \\
-t_{3}+i G^{0}
\end{array}\right)
$$

because, in general,

$$
w \neq U_{v} w^{\prime},
$$

such as for $U_{v}=e^{i \theta \sigma_{1} / 2}$.

The solution is to promote Eq. (24) to define the real fields $t_{i}$ as

$$
t_{i} \equiv \frac{2}{v} s_{i}, \quad i=1,2,3 .
$$

The definition of Eq. (50) ensures that $t_{i}$ would transform as vectors under $S O(3)_{H}$ when $S U(2)_{H}$ transformations are applied to $w$ and $\langle w\rangle$ in the definition (42) of $s_{i}$. The $s_{0}$ component depends on $t_{i}$ by the basis invariant relation

$$
s_{0}=\frac{v}{2}\langle\hat{\mathbf{r}}\rangle \cdot \mathbf{t},
$$

where $\langle\hat{\mathbf{r}}\rangle$ is the unit vector in the direction of $\langle\mathbf{r}\rangle$. The relation (51) is proved in appendix $\mathrm{C}$.

To write the second and third terms of Eq. (41) in terms of $t_{i}$ it is necessary to find the parametrization of $w$ in terms of $t_{i}$ and $G^{0}$. The desired covariant relation is

$$
w=\frac{1}{\sqrt{2}}\left(i G^{0} \mathbb{1}_{2}+t_{i} \sigma_{i}\right)\langle\hat{w}\rangle,
$$

where $\langle\hat{w}\rangle=\langle w\rangle /|\langle w\rangle|$. One can confirm that Eq. (52) satisfies Eq. (50) using Eq. (13). The covariance can be also checked,

$$
w^{\prime}=U w=\frac{1}{\sqrt{2}}\left(i G^{0} \mathbb{1}_{2}+t_{i}^{\prime} \sigma_{i}\right)\left\langle\hat{w}^{\prime}\right\rangle,
$$

where

$$
t_{i}^{\prime}=R_{i j} t_{j}
$$

and $R_{i j}$ is related to $U$, a transformation in $S U(2)_{H}$, satisfying Eq. (8). The dependence on $G^{0}$ is fixed by imposing that Eq. (52) reduces to Eq. (17) in the PCH basis. Notice Eq. (52) differs from Eq. (46) of Ref. [6] as the fields $t_{i}$ transform as vectors under $S O(3)_{H}$.

We can thus rewrite the second term of Eq. (41) in terms of $t_{i}$ using Eqs. (46) and (52) as

$$
w^{\dagger}\langle\mathbf{M}\rangle w=\frac{m^{2}}{2}\left[\mathbf{t}^{2}-(\langle\hat{\mathbf{r}}\rangle \cdot \mathbf{t})^{2}\right]
$$

The third term of Eq. (41) can be also easily rewritten in terms of $t_{i}$ by using Eq. (50). The sum of the second and the third terms of Eq. (41) defines the mass matrix for the physical neutral scalars by

$$
w^{\dagger}\langle\mathbb{M}\rangle w+\frac{1}{2} \Lambda_{\mu \nu} s_{\mu} s_{\nu}=\frac{1}{2}\left(\mathcal{M}_{N}\right)_{i j} t_{i} t_{j},
$$

giving the basis covariant relation 


$$
\begin{aligned}
\mathcal{M}_{N}= & m^{2}\left[\mathbb{1}_{3}-\frac{\langle\mathbf{r}\rangle\langle\mathbf{r}\rangle^{\top}}{\mid\left\langle\left.\mathbf{r}\right|^{2}\right.}\right]+\mid\langle\mathbf{r}\rangle\left[\left[\tilde{\Lambda}+\Lambda_{00} \frac{\langle\mathbf{r}\rangle\langle\mathbf{r}\rangle^{\top}}{|\langle\mathbf{r}\rangle|^{2}}\right]\right. \\
& +\langle\mathbf{r}\rangle \boldsymbol{\Lambda}_{\mathbf{0}}^{\top}+\boldsymbol{\Lambda}_{\mathbf{0}}\langle\mathbf{r}\rangle^{\top},
\end{aligned}
$$

where $|\langle\mathbf{r}\rangle|=v^{2} / 4$. Equation (57) is the generalization of Eq. (25) for an arbitrary basis.

Equation (57) illustrates two points: (i) the whole potential after EWSB can be completely defined in terms of the set

$$
\left\{m^{2}, \Lambda_{00}, \Lambda_{0},\langle\mathbf{r}\rangle, \mathcal{M}_{N}\right\},
$$

in an arbitrary basis, since $\tilde{\Lambda}$ can be written in terms of the set. Moreover, the objects in the set have the same transformation properties under the reparametrization group $S U(2)_{H}$ as the set $\left\{M_{0}, \Lambda_{00}, \Lambda_{0}, \mathbf{M}, \tilde{\Lambda}\right\}$ that defines the potential before EWSB [19]: two scalars [26], two vectors and one rank-2 tensor. (ii) We can define a physical neutral Higgs (PNH) basis, in contrast to the $\mathrm{PCH}$ basis, being the basis where $\mathcal{M}_{N}$ is diagonal. In general, this basis will coincide neither with the $\mathrm{PCH}$ basis nor with the basis with diagonal $\tilde{\Lambda}$ (the canonical $C P$ basis in Ref. [19]).

The relations (26) and (31) can be written in the basis covariant form

$$
\begin{aligned}
Y= & \frac{1}{2}\left[m^{2}-\left\langle r_{0}\right\rangle \Lambda_{00}-\boldsymbol{\Lambda}_{\mathbf{0}} \cdot\langle\mathbf{r}\rangle\right]\left(\mathbb{1}_{2}-\sigma_{i}\left\langle\hat{r}_{i}\right\rangle\right) \\
& -\frac{1}{2} \sigma_{i}\left(\mathcal{M}_{N}\right)_{i j}\left\langle\hat{r}_{j}\right\rangle .
\end{aligned}
$$

We made use of the relation

$$
\mathbb{1}_{2}-\langle\hat{w}\rangle\langle\hat{w}\rangle^{\dagger}=\frac{1}{2}\left[\mathbb{1}_{2}-\sigma_{i}\left\langle\hat{r}_{i}\right\rangle\right] .
$$

One can check Eq. (59) reduces to Eqs. (26) and (31) in the $\mathrm{PCH}$ basis. It is also important to remark that $Y$ in Eq. (59) is independent on the particular VEV. For a different minimum of the same potential (or extremum if we do not require positive definite $\mathcal{M}_{N}$ and $m^{2}$ ), $\mathcal{M}_{N}, m^{2}$, and $\langle\mathbf{r}\rangle$ differ in such a way that $Y$ is the same. In addition, we can write the $(i j)=(33)$ component of Eq. (26) in the following basis covariant form

$$
\langle\hat{w}\rangle^{\dagger} Y\langle\hat{w}\rangle=-\frac{1}{2}\left\langle\hat{r}_{i}\right\rangle\left(\mathcal{M}_{N}\right)_{i j}\left\langle\hat{r}_{j}\right\rangle .
$$

To obtain the interaction terms [25]

$$
\begin{gathered}
\left.V_{3}\right|_{\mathrm{SSB}}=\Lambda_{\mu \nu} s_{\mu} r_{\nu}, \\
\left.V_{4}\right|_{\mathrm{SSB}}=\frac{1}{2} \Lambda_{\mu \nu} r_{\mu} r_{\nu},
\end{gathered}
$$

in terms of the real fields $t_{i}$, we should calculate $r_{\mu}$ in Eq. (4) using Eq. (50). Splitting

$$
r_{\mu}=x_{\mu}+y_{\mu},
$$

where

$$
\begin{aligned}
& x_{\mu}=\frac{1}{2} u^{\dagger} \sigma_{\mu} u, \\
& y_{\mu}=\frac{1}{2} w^{\dagger} \sigma_{\mu} w,
\end{aligned}
$$

we obtain the following covariant relations for $y$,

$$
y_{0}=\frac{1}{4}\left[\left(G^{0}\right)^{2}+\mathbf{t}^{2}\right]
$$

$$
y_{i}=\frac{1}{4}\left[\left(G^{0}\right)^{2}-\mathbf{t}^{2}\right]\left\langle\hat{r}_{i}\right\rangle+\frac{1}{2}(\langle\hat{\mathbf{r}}\rangle \cdot \mathbf{t}) t_{i}+\frac{1}{2} G^{0}(\mathbf{t} \times\langle\hat{\mathbf{r}}\rangle)_{i} .
$$

Since each component of $u=\left(u_{1}, u_{2}\right)^{\top}$ is a combination of the physical charged Higgs $h^{+}$and the charged Goldstone $G^{+}$, there is no need to write them in terms of real fields. The expression (65) can be kept as the covariant relation.

A last observation about Eq. (68) concerns the transformation properties of $G^{0}$ under refections, i.e., $C P$ symmetry. To keep the transformation properties of the last term of Eq. (68) to be the same as the preceding terms we conclude that $G^{0}$ should be a pseudoscalar (scalar under $S O(3)_{H}$ and changing sign under reflection or $C P$ ) and consequently $C P$-odd irrespective of the $C P$ properties of the potential. (See appendix D of Ref. [6].)

\section{PHYSICAL BASIS (P-BASIS)}

The physical basis (P-basis) should be defined as the basis where all the fields possess definite masses. From Sec. II, we conclude that the mass matrix for physical neutral scalars $\left(\mathcal{M}_{N}\right)$ in the PCH basis will be not diagonal in general. From Sec. III, the basis where $\mathcal{M}_{N}$ is diagonal (PNH basis) would mix the physical charged Higgs $h^{+}$ with the charged Goldstone $G^{+}$. Thus, neither the PCH basis nor the PNH basis coincide with the physical basis.

To achieve the P-basis, we need independent basis transformations on the upper $(u)$ and lower components $(w)$ of the doublets, i.e.,

$$
u \rightarrow U_{u} u, \quad w \rightarrow U_{w} w,
$$

where $U_{u}, U_{w}$ are different transformations in $S U(2)$. The transformations in Eq. (69) are legitimate basis transformations only after EWSB since they still preserve the EM symmetry, for a neutral vacuum, but do not preserve the $S U(2)_{L} \otimes U(1)_{Y}$ gauge structure of the doublets, except for $U_{u}=U_{w}$. The general group of basis transformations generated by Eq. (69) is $S U(2) \otimes S U(2)$ instead of $S U(2)_{H}$ valid before EWSB.

The P-basis can be achieved either from the $\mathrm{PCH}$ basis or from the PNH basis. The latter choice is more convenient. Let us choose the PNH basis for which

$$
\mathcal{M}_{N}=\operatorname{diag}\left(m_{1}^{2}, m_{2}^{2}, m_{3}^{2}\right) .
$$

The VEV $\langle w\rangle$ will be in the general form of Eq. (38), different from the PCH basis. The respective $\left\langle r_{\mu}\right\rangle$ would be parametrized in the form of Eq. (39).

In the PNH basis, the fields $t_{i}$ and $G^{0}$, contained in $w$, already have definite masses. The components of $u$, however, are combinations of the physical charged fields $h^{+}$ and $G^{+}$. The relation between $u$ and the physical fields is 
given by

$$
u=U_{v} u^{\prime},
$$

where $u^{\prime}$ refers to $u$ in the PCH basis,

$$
u^{\prime}=\left(h^{+}, G^{+}\right)^{\top} .
$$

In a basis invariant form, we know the component of $u$ parallel to $\langle w\rangle$ is the charged Goldstone,

$$
\langle\hat{w}\rangle^{\dagger} u=G^{+} .
$$

The orthogonal direction contains the physical $h^{+}$.

Obviously, the quadratic part of the potential after EWSB will be

$$
\left.V_{2}\right|_{\mathrm{SSB}}=m^{2} h^{+} h^{-}+\frac{1}{2} m_{i}^{2} t_{i}^{2}
$$

The remaining task to completely define the potential after EWSB is to write the interaction terms in Eqs. (62) and (63) in terms of $\left\{t_{i}, G^{0}, h^{+}, G^{+}\right\}$. The sole dependence of those interaction terms on $u$ comes from $x_{\mu}$ in Eq. (65). The component $x_{0}$ is basis independent and can be readily written

$$
x_{0}=\frac{1}{2}\left(G^{-} G^{+}+h^{-} h^{+}\right) .
$$

The spatial components can be written

$$
x_{i}=\left(\tilde{R}_{v}\right)_{i j} x_{j}^{\prime}
$$

where $\left(\tilde{R}_{v}\right)_{i j}=\operatorname{Tr}\left[U_{v}^{\dagger} \sigma_{i} U_{v} \sigma_{j}\right]$ and

$$
\begin{gathered}
x_{1}^{\prime}=\frac{1}{2}\left(h^{-} G^{+}+h^{+} G^{-}\right), \\
x_{2}^{\prime}=\frac{-i}{2}\left(h^{-} G^{+}-h^{+} G^{-}\right), \\
x_{3}^{\prime}=\frac{1}{2}\left(G^{-} G^{+}-h^{-} h^{+}\right),
\end{gathered}
$$

The variables $s_{\mu}$ can be written in terms of $t_{i}$ using Eqs. (50) and (51) while the variables $y_{\mu}$ are defined in Eqs. (67) and (68).

\section{DISCUSSIONS}

Equation (61) relates the depth of the potential in the minimum with the mass matrix of the physical neutral scalars. We can obtain bounds on the depth of the potential from the relations

$$
V(\langle r\rangle)=\frac{1}{2} V_{2}(\langle r\rangle)=-V_{4}(\langle r\rangle),
$$

where $\langle r\rangle$ represents an extremum while $V_{2}$ and $V_{4}$ refer, respectively, to the quadratic and quartic part of the potential before EWSB, defined in Eqs. (2) and (3), evaluated in the extremum. The first equality of Eq. (80) can be written using Eq. (61) as

$$
V(\langle r\rangle)=-\frac{1}{2}\left\langle r_{0}\right\rangle\left\langle\hat{r}_{i}\right\rangle^{\top}\left(\mathcal{M}_{N}\right)_{i j}\left\langle\hat{r}_{j}\right\rangle .
$$

From the relation above, we can deduce the following bounds for the depth of a minimum $\langle r\rangle$,

$$
-\frac{1}{2}\left\langle r_{0}\right\rangle m_{3}^{2} \leq V(\langle r\rangle) \leq-\frac{1}{2}\left\langle r_{0}\right\rangle m_{1}^{2},
$$

where $m_{3}^{2}$ and $m_{1}^{2}$ are, respectively, the greatest and the least eigenvalue of $\mathcal{M}_{N}$. We can conclude that a minimum will be deeper if the respective masses for the neutral scalars and the value of $v$ are greater.

A different physical bound can be extracted from condition (B11) necessary for bounded below potentials and from the positive definiteness of $\mathcal{M}_{N}$. From Eq. (57) and $e_{\perp}^{\top}\left(\tilde{\Lambda}+\Lambda_{00} \mathbb{1}\right) e_{\perp}>0$ we arrive at

$$
m^{2}-m_{3}^{2}<\frac{v^{2}}{4} \Lambda_{00}
$$

where $e_{\perp}$ is any unit vector orthogonal to $\langle\hat{\mathbf{r}}\rangle$. The last inequality means the mass of the charged Higgs can not be arbitrarily large compared to the masses of the neutral scalars.

In Sec. III we have found the set of Eq. (58) could be chosen as the physical parameters that define the 2HDM potential with a nontrivial vacuum. Among the elements of the set, it is clear that the masses are physical observables. On the other hand, the connection of the coupling constants and mixing matrices appearing in the interaction terms with physical observables is not direct. For example, devising scattering observables to extract the three parameters composing $\Lambda_{0}$, present in $V_{3}$ and $V_{4}$, does not seem a straightforward task. The form of $V_{3}$ in terms of the physical fields, given in appendix E, reinforce such difficulty. The explicit form of $V_{3}$ and $V_{4}$ in the physical basis can be also found in Eqs. (57)-(60) of Ref. [6], although the dependence on the mass matrix of the neutral scalars are not explicitly shown. An attempt to extract the observable parameters in the $2 \mathrm{HDM}$, aiming to identify the presence of discrete symmetries through measurements, was made in Ref. [27]. Nevertheless, separating the set of Eq. (58) and finding the relation of other parameters with the set is important to establish the number of independent parameters possible. The violation of any relation between parameters would indicate a model with a scalar sector distinct of the 2HDM. These relations should be constrained by experimental data and studies of the bounds on the mass of the physical charged Higgs $[28,29]$ or of the decay width of the physical Higgs bosons [2,30] already exist in the literature. Of course, higher order effects, such as the exchange of quarks, would modify these tree-level relations. The number of minima may be also modified when higher order contributions are taken into account. The existence of at most two minima, for example, may not be true beyond tree level [31].

Another aspect of the identification of physical parameters concerns the remaining reparametrization freedom such as the one in Eq. (32). That rephasing transformation freedom is particularly important when counting the number of parameters of the mixing matrix $\tilde{R}_{v}$ in Eq. (76). 
Since $\tilde{R}_{v}$ appears in the couplings involving the physical charged fields it may seem that it is a physical rotation matrix, needing three angles for its parametrization. However, only two angles are physical. The reason is that the reminiscent reparametrization freedom induced by Eq. (32) can remove one angle. Such reparametrization freedom is equivalent to rotations around $\langle\mathbf{r}\rangle$. An explicit parametrization using two angles is available in Eqs. (E8) and (E9) of appendix E.

The case of $C P$ conserving potentials includes the MSSM 2HDM potential (see Ref. [2]) and can be easily analyzed by setting $\Lambda_{2 i}=0$ for $i \neq 2$ and $M_{2}=0$ (or real $Y)$. In addition, if there is no SPCV, we have $\left\langle r_{2}\right\rangle=0$. In this case, from Eq. (57), we see the neutral scalar $t_{2}$ does not mix with other scalars and corresponds to a $C P$-odd field with mass

$$
m_{2}^{2}=m^{2}+\frac{v^{2}}{4} \Lambda_{22}
$$

Relation (84) is equivalent to a known relation encountered in the MSSM (see Eq. (10) of Ref. [11]), where the pseudoscalar $t_{2}$ is usually called $A$ and $\frac{v^{2}}{4} \Lambda_{22}=-m_{W}^{2}$. The remaining neutral scalars $t_{1}, t_{3}$ are $C P$-even and their mass matrix can be also read from Eq. (57).

It is important to stress that the original basis transformations valid before EWSB forming the $S U(2)_{H}$ group could be explicitly transposed to the fields after EWSB. Although the possibility of transposition could be foreseen, various properties of the transformations after EWSB could not be anticipated. For example, the basis transformations after EWSB mix the physical charged Higgs $h^{+}$ with the charged Goldstone while the neutral fields $t_{i}$ mix among them [through the same $S U(2)_{H} \sim S O(3)_{H}$ ] without mixing with the neutral Goldstone that transforms as a scalar of $S U(2)_{H}$.

From the discussions of Sec. IV, we can see there is an important distinction between basis transformation and reparametrization. The transformations of Eq. (69) constitute legitimate basis transformations that preserve the gauge structure after EWSB but they do not configure as reparametrization transformations. On the one hand, only the original $S U(2)_{H}$ basis transformations that preserve the $S U(2)_{L}$ gauge structure configure as reparametrization transformations. On the other hand, the maximal semisimple group of transformations which mix four real fields, $t_{i}$ and $G^{0}$, is $S O(4)$. In addition, if we do not impose the kinetic part to be invariant, the reparametrization group $S U(2)_{H}$ can be extended to $S L(2, c)$ [9].

As a terminological issue, the term basis transformations (or horizontal transformations) should be accompanied by the gauge structure that they preserve, to be precise. For example, for the 2HDM treated here, it is important to specify if the basis transformations act before $\left[S U(2)_{L} \otimes\right.$ $\left.U(1)_{Y}\right]$ or after EWSB $\left[U(1)_{\mathrm{EM}}\right]$.
In general, the horizontal group after SSB will be larger than the horizontal group before SSB. It should be remarked that usually the physical mixing parameters belong to the additional basis transformations only allowed after SSB. For example, the CKM matrix for quarks comes from the difference between the rotations on the fields $\left\{u_{L}, c_{L}, t_{L}\right\}$ and $\left\{d_{L}, s_{L}, b_{L}\right\}$ necessary to diagonalize the respective mass matrices; applying basis transformations before EWSB, it is only possible to diagonalize one of the up or down quark Yukawa coupling matrices. A similar structure appears in 2HDMs for which the mixing among neutral scalars, the matrix $\tilde{R}_{v}$, appears as the difference between the $\mathrm{PCH}$ basis and the $\mathrm{PNH}$ basis.

For general $N$-Higgs-doublet models (NHDMs), the covariant relation for the mass matrix of neutral scalars can be easily written by generalizing Eqs. (52) and (57) to $N$ Higgs doublets. The covariant relation for the mass matrix of charged scalars $\langle\mathbb{M}\rangle$ [32] was found in Ref. [25]. The fields $t_{i}$, however, will transform as a vector of $\operatorname{adj} S U(N)_{H}$, living in a real vector space of $N^{2}-1$ dimensions. Since, in general, a transformation in $S O\left(N^{2}-1\right)$, a larger group than $\operatorname{adj} S U(N)_{H}$, will be required to diagonalize $\mathcal{M}_{N}$, the PNH basis can not be reached by reparametrization but only by general horizontal transformations valid after EWSB. The corresponding basis transformation group will be $S O\left(N^{2}-1\right) \otimes S U(N)$, the first factor acting on the neutral scalars and the second on the charged scalars independently. The enlargement of the basis transformation group after EWSB compared to the basis transformation group before EWSB is greater in NHDMs, with $N>2$, than in the two-Higgs doublet case $(N=2)$. But the difference is not just quantitative. For the 2HDM potential, the basis transformation group after EWSB, $S U(2) \otimes S U(2)$, is just the double of the basis transformation group $S U(2)_{H}$ before EWSB, which can be understood as the original basis transformation acting independently on the upper $u$ and lower $w$ components of the doublets, as described in Eq. (69). For $N>2$, the factor $S O\left(N^{2}-1\right)$ necessary to diagonalize $\mathcal{M}_{N}$ and, consequently, necessary to reach the physical basis, cannot be thought of as the original reparametrization group $S U(N)_{H}$ acting independently on the lower components $w$ of the doublets.

Finally, we can say that a nontrivial horizontal structure in the scalar sector of a theory enriches the latter significantly, opening the possibility of different phenomenology such as different symmetry breaking patterns. At the same time, the theory becomes less predictive as many more free parameters are available. Nevertheless, useful physical information can be extracted from the horizontal structure by classifying the transformation properties of the parameters appearing in the potential. These properties constrain the relations between parameters before and after SSB, relating, for instance, vectors of the horizontal group with vectors. In the $2 \mathrm{HDM}$ potential analyzed here, we could relate, for example, the rank- 2 tensor $\tilde{\Lambda}$, appearing 
before EWSB, with the mass matrix of the neutral scalars $\mathcal{M}_{N}$, only extractable after EWSB. Moreover, these relations were basis invariant. It is important to notice that the transformation properties of the parameters refer to the horizontal group $S U(2)_{H}$ acting on the Higgs doublets before EWSB. Although the horizontal group acting on the fields after EWSB could be larger, the transformation properties of the parameters followed essentially from the original horizontal group valid before EWSB. Obviously, a transformation in the enlarged horizontal group is usually necessary to reach the physical basis where all fields have definite masses.

\section{ACKNOWLEDGMENTS}

The author would like to thank Igor Ivanov for usefull discussions. This work was supported by Fundação de Amparo à Pesquisa do Estado de São Paulo (Fapesp).

\section{APPENDIX A: EXTREMUM EQUATIONS}

Any neutral extremum $\left\langle r_{\mu}\right\rangle$ of the potential in Eq. (1) should satisfy the following extremum equations [25]

$$
\begin{gathered}
M_{0}+\Lambda_{00}\left\langle r_{0}\right\rangle+\Lambda_{\mathbf{0}} \cdot\langle\mathbf{r}\rangle=m^{2}, \\
M_{i}+\Lambda_{i 0}\left\langle r_{0}\right\rangle+\tilde{\Lambda}_{i j}\left\langle r_{j}\right\rangle=-m^{2}\left\langle\hat{r}_{i}\right\rangle,
\end{gathered}
$$

where $i=1,2,3$ and $m^{2}$ is the mass squared of the physical charged Higgs. The minus sign on the righthand side of Eq. (A2) is reminiscent of the Minkowski metric adopted in Ref. [25].

The original extremum equation on the doublets reads

$$
\langle\mathbb{M}\rangle\langle w\rangle=0,
$$

for $\langle u\rangle=0$ and $\langle w\rangle \neq 0$. Equation (A3) means $\langle w\rangle$ is an eigenvector of $\langle\mathbb{M}\rangle$ with null eigenvalue.

\section{APPENDIX B: BOUNDED BELOW CONDITION}

We seek here the necessary conditions for a bounded below potential using a method distinct to the ones adopted in Refs. [3,9]. We will restrict ourselves to positive definite $V_{4}$.

Rewriting $V_{4}$ for $r_{0}=|\mathbf{r}|$ we obtain

$$
V_{4}=\frac{1}{2} \mathbf{r}^{\top}\left(\tilde{\Lambda}+\Lambda_{00} \mathbb{1}_{3}\right) \mathbf{r}+|\mathbf{r}| \mathbf{r} \cdot \Lambda_{\mathbf{0}} .
$$

All variables $r_{i}$ will be treated here as c-numbers. We seek the direction $\mathbf{r}$ for which the potential increases more slowly. We minimize then

$$
V_{4}^{\prime}=V_{4}+\frac{1}{2} \lambda\left(\mathbf{r}^{2}-1\right)
$$

constraining $\mathbf{r}$ to be in the unit sphere using the Lagrange multiplier method.
Differentiating,

$$
\begin{gathered}
\frac{\partial V_{4}^{\prime}}{\partial r_{i}}=\tilde{\Lambda}_{i j} r_{j}+\Lambda_{00} r_{i}+\Lambda_{0 i}|\mathbf{r}|+\hat{r}_{i}\left(\mathbf{r} \cdot \Lambda_{\mathbf{0}}\right)+\lambda r_{i}, \\
\frac{\partial V_{4}^{\prime}}{\partial \lambda}=\frac{1}{2}\left(\mathbf{r}^{2}-1\right) .
\end{gathered}
$$

Equation (B3) yields

$$
\hat{\mathbf{r}}=-\left[\tilde{\Lambda}+\left(\Lambda_{00}+\lambda+\hat{\mathbf{r}} \cdot \boldsymbol{\Lambda}_{\mathbf{0}}\right) \mathbb{1}_{3}\right]^{-1} \boldsymbol{\Lambda}_{\mathbf{0}} .
$$

The values of $\hat{\mathbf{r}} \cdot \boldsymbol{\Lambda}_{\mathbf{0}}$ corresponding to an extremum is given by the roots of

$$
\hat{\mathbf{r}} \cdot \Lambda_{\mathbf{0}}=f\left(\hat{\mathbf{r}} \cdot \Lambda_{\mathbf{0}}+\lambda\right),
$$

constrained by

$$
\frac{d f(x)}{d x}=1,
$$

for $x=\hat{\mathbf{r}} \cdot \boldsymbol{\Lambda}_{\mathbf{0}}+\lambda$. The function $f(x)$ is defined by

$$
f(x) \equiv-\boldsymbol{\Lambda}_{\mathbf{0}}^{\top}\left[\tilde{\Lambda}+\left(\Lambda_{00}+x\right) 1_{3}\right]^{-1} \boldsymbol{\Lambda}_{\mathbf{0}} .
$$

Equation (B6) is found by projecting Eq. (B5) to $\boldsymbol{\Lambda}_{\mathbf{0}}$ while Eq. (B7) is equivalent to the requirement $\hat{\mathbf{r}} \cdot \hat{\mathbf{r}}=1$. The components of $\hat{\mathbf{r}}$ perpendicular to $\boldsymbol{\Lambda}_{\mathbf{0}}$ can be found from Eq. (B5) once $\hat{\mathbf{r}} \cdot \boldsymbol{\Lambda}_{\mathbf{0}}$ is known.

For any extremum satisfying Eq. (B5) we find for Eq. (B1) the value

$$
\left.V_{4}\right|_{\text {extremum }}=\left.\frac{1}{2} \mathbf{r}^{2}(-\lambda)\right|_{\text {extremum. }} .
$$

We see all the Lagrange multipliers $\lambda$ corresponding to an extremum should be negative. In particular, the greatest of them should be negative.

In the basis for which $\tilde{\Lambda}+\Lambda_{00} \mathbb{1}_{3}=\operatorname{diag}\left(a_{1}, a_{2}, a_{3}\right)$, $a_{1}>a_{2}>a_{3}$,

$$
f(x)=-\sum_{i=1}^{3} \frac{\Lambda_{0 i}^{2}}{a_{i}+x} .
$$

A plot of $f(x)$, with $a_{i}>0$, can be seen in Fig. 1 jointly with the solution of greatest $\lambda$. We see there are at least two extrema corresponding to the least and greatest $\lambda$. The intermediary extrema may not exist depending on the minimum slope of the curves. For example, in Fig. 1, for $-a_{2} \leq x \leq-a_{3}$, there is no solution for $f^{\prime}(x)=1$.

From the schematic view of Fig. 1 we see $a_{i}>0$ is necessary to have positive $-\lambda$ and consequently positive definite $V_{4}$, unless $\Lambda_{0 i}=0$ for nonpositive $a_{i}$. In a general basis, it is necessary that

$$
\Lambda_{00}+\text { eigenvalues }(\tilde{\Lambda})>0,
$$

unless $\boldsymbol{\Lambda}_{\mathbf{0}}$ have null projection in some eigenvector direction. To assure $V_{4}$ is positive definite, it is necessary and sufficient to have the greatest Lagrange multiplier

$$
\max \lambda<0 .
$$




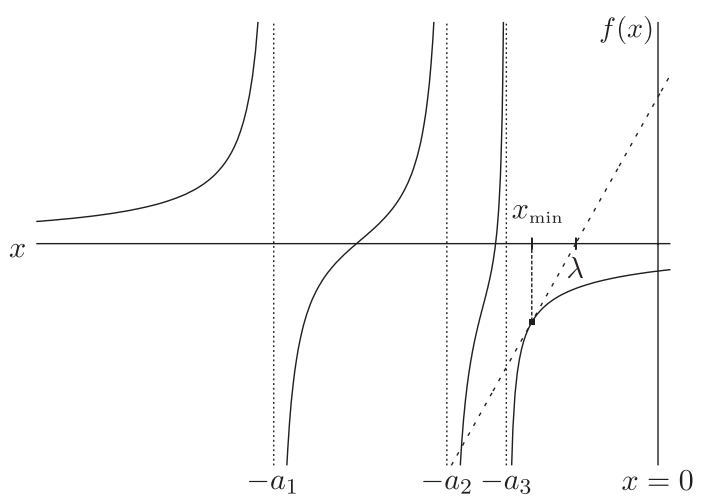

FIG. 1. Plot of typical $f(x)$. The dot lies at $\left(x_{\min }, f\left(x_{\min }\right)\right)$ where $x_{\min }$ is the greatest value that satisfies $f^{\prime}(x)=1$. Equation (B6) defines the value of $\lambda$ depicted as the intersection of the line $x-x_{\min }+f\left(x_{\min }\right)$ with the $x$-axis.

From Eq. (B6), the distance between the greatest $x_{\min }$ and the greatest $\lambda$ is $\left|\hat{\mathbf{r}} \cdot \boldsymbol{\Lambda}_{\mathbf{0}}\right|=-\hat{\mathbf{r}} \cdot \boldsymbol{\Lambda}_{\mathbf{0}}$.

Finally, if $a_{i}>0$ and one makes $\left|\Lambda_{0 i}\right|$ small enough, we can always find $\lambda<0$, proving the assertion preceding Eq. (34). As $\left|\Lambda_{0 i}\right|$ get smaller, the curves of $f(x)$ get closer to the $x$-axis. In special, from $f(x) \geq\left. f(x)\right|_{a_{1}, a_{2} \rightarrow a_{3}}, a_{3}=$ $\min \left(a_{i}\right)$, we can conclude that

$$
2\left|\boldsymbol{\Lambda}_{0}\right|<a_{3}
$$

is a sufficient condition.

For $\left|r_{0}\right|>|\mathbf{r}|$ we can parametrize $r_{0}=e^{\chi}|\mathbf{r}|, \chi>0$. The analysis of the minimization of $V_{4}$ for fixed $|\mathbf{r}|$ is equivalent to the preceding analysis replacing $\Lambda_{00} \rightarrow$ $\Lambda_{00} e^{2 \chi}$ and $\Lambda_{0 i} \rightarrow \Lambda_{0 i} e^{\chi}$. If $\left|\Lambda_{0}\right| / \Lambda_{00} \leq 1$, condition (B13) is preserved for $\chi>0$ once it is valid for $\chi=0$. If $\left|\boldsymbol{\Lambda}_{\mathbf{0}}\right| / \Lambda_{00}>1$ and $\lambda_{i}>0$, a sufficient condition is

$$
\frac{\left|\Lambda_{0}\right|^{2}}{\Lambda_{00}}<\min \left(\lambda_{i}\right)
$$

where $\lambda_{i}$ are the eigenvalues of $\tilde{\Lambda}$.

\section{APPENDIX C: PROOF OF EQ. (51)}

From the completeness of the $\sigma_{\mu}$ matrices [33],

$$
\frac{1}{2}\left(\sigma_{\mu}\right)_{a b}\left(\sigma_{\mu}\right)_{c d}=\delta_{a d} \delta_{c b},
$$

we can calculate, for a neutral vacuum $\langle w\rangle \neq 0$,

$$
\begin{aligned}
\left\langle r_{0}\right\rangle s_{0}+\left\langle r_{i}\right\rangle s_{i} & =\frac{1}{4} \operatorname{Tr}\left[\langle w\rangle\langle w\rangle^{\dagger} \sigma_{\mu}\right] \operatorname{Tr}\left[\sigma_{\mu}\left(w\langle w\rangle^{\dagger}+\text { H.c. }\right)\right] \\
& =\frac{1}{2} \operatorname{Tr}\left[\left(w\langle w\rangle^{\dagger}+\text { H.c. }\right)\langle w\rangle\langle w\rangle^{\dagger}\right]=2\left\langle r_{0}\right\rangle s_{0},
\end{aligned}
$$

where $\left\langle r_{0}\right\rangle=|\langle w\rangle|^{2} / 2=v^{2} / 4$. Hence,

$$
s_{0}=\left\langle\hat{r}_{i}\right\rangle s_{i}
$$

since $\left\langle r_{0}\right\rangle=\sqrt{\left\langle r_{i}\right\rangle\left\langle r_{i}\right\rangle}$. With the same reasoning, one can prove

$$
s_{0} y_{0}=s_{i} y_{i} \text {. }
$$

\section{APPENDIX D: BASIS COVARIANCE FOR $\langle M\rangle$}

It is important to stress that the definition of the charged mass matrix $\langle\mathbb{M}\rangle$ is covariant by basis transformation (44) in the following sense. The definition of the charged mass matrix in Eq. (14) is valid in any basis, in particular, in the $\mathrm{PCH}$ basis,

$$
\operatorname{diag}\left(m^{2}, 0\right)=\left\langle\mathbb{M}^{\prime}\right\rangle=Y^{\prime}+\frac{1}{2} \sigma_{\mu} \Lambda_{\mu \nu}^{\prime}\left\langle r_{\nu}^{\prime}\right\rangle .
$$

The Eq. (43) can thus be written as

$$
U_{v}^{\dagger}\left(Y+\frac{1}{2} \sigma_{\mu} \Lambda_{\mu \nu}\left\langle r_{\nu}\right\rangle\right) U_{v}=Y^{\prime}+\frac{1}{2} \sigma_{\mu} \Lambda_{\mu \nu}^{\prime}\left\langle r_{\nu}^{\prime}\right\rangle,
$$

where the relation between $\left\{Y^{\prime}, \Lambda^{\prime},\left\langle r^{\prime}\right\rangle\right\}$ in the PCH basis and $\{Y, \Lambda,\langle r\rangle\}$ in the original basis is

$$
\begin{gathered}
Y^{\prime}=U_{v}^{\dagger} Y U_{v}, \\
\left\langle r_{\mu}^{\prime}\right\rangle=R_{\mu \nu}^{\top}\left\langle r_{\nu}\right\rangle, \\
\Lambda_{\mu \nu}^{\prime}=R_{\mu \alpha}^{\top} \Lambda_{\alpha \beta} R_{\beta \nu},
\end{gathered}
$$

and $R_{\mu \nu}=R_{\mu \nu}\left(U_{v}\right)$ is given by Eq. (40). Hence, the first term of (41) is form invariant,

$$
u^{\dagger}\langle\mathbb{M}\rangle u=u^{\prime \dagger}\left\langle\mathbb{M}^{\prime}\right\rangle u^{\prime}
$$

\section{APPENDIX E: INTERACTION TERMS}

The interaction terms can be simplified into

$$
\begin{aligned}
\left.\frac{v}{2} V_{3}\right|_{\mathrm{SSB}}= & -\left[u^{\dagger} Y u+w^{\dagger} Y w\right] t_{\|}+m^{2}\left[x_{0} t_{\|}-\mathbf{x} \cdot \mathbf{t}\right] \\
& +\left\langle r_{0}\right\rangle \boldsymbol{\Lambda}_{\mathbf{0} \perp} \cdot \mathbf{t}_{\perp}\left[\left|u_{\perp}\right|^{2}+\frac{1}{2} \mathbf{t}_{\perp}^{2}\right] \\
& +(\mathbf{x}+\mathbf{y})^{\top} \mathcal{M}_{N} \mathbf{t},
\end{aligned}
$$

$$
\begin{aligned}
\left.V_{4}\right|_{\mathrm{SSB}}= & \frac{1}{2} \Lambda_{00}\left(r_{0}^{2}-\mathbf{r}^{2}\right)+\left(r_{0}-\langle\hat{\mathbf{r}}\rangle \cdot \mathbf{r}\right) \boldsymbol{\Lambda}_{\mathbf{0}} \cdot \mathbf{r}+\frac{\mathbf{r}^{\top} \mathcal{M}_{N} \mathbf{r}}{2\left\langle r_{0}\right\rangle} \\
& +\frac{1}{2}\left(\Lambda_{00}-\frac{m^{2}}{\left\langle r_{0}\right\rangle}\right) \mathbf{r}_{\perp}^{2},
\end{aligned}
$$

where

$$
\begin{gathered}
u_{\perp} \equiv u-\langle\hat{w}\rangle\langle\hat{w}\rangle^{\dagger} u, \\
t_{\|} \equiv \mathbf{t} \cdot\langle\hat{\mathbf{r}}\rangle, \\
\mathbf{t}_{\perp} \equiv \mathbf{t}-\langle\hat{\mathbf{r}}\rangle t_{\|}, \\
\left|u_{\perp}\right|^{2}=x_{0}-\mathbf{x} \cdot\langle\hat{\mathbf{r}}\rangle,
\end{gathered}
$$




$$
\mathbf{t}_{\perp}^{2}=2\left(y_{0}-\mathbf{y} \cdot\langle\hat{\mathbf{r}}\rangle\right), \quad \text { (E7) } \quad \text { where }
$$

and $\boldsymbol{\Lambda}_{\mathbf{0} \perp}$ is analogous to $\mathbf{t}_{\perp}$.

One can also explicit the matrices $U_{v}$ and $\tilde{R}\left(U_{v}\right)$ in

$$
U(\theta, \varphi) \equiv e^{-i(1 / 2) \sigma_{3} \varphi} e^{-i(1 / 2) \sigma_{2} \theta} .
$$

Eqs. (71) and (76) choosing an explicit parametrization:

$$
\begin{gathered}
U_{v}=U\left(\theta_{v}, \xi\right) i \sigma_{2}, \\
\tilde{R}_{v}=\tilde{R}\left(\theta_{v}, \xi\right) \tilde{R}(\pi, 0)=\tilde{R}\left(U_{v}\right)
\end{gathered}
$$

$$
\tilde{R}(\theta, \varphi) \equiv e^{-i J_{3} \varphi} e^{-i J_{2} \theta} .
$$

The generators of rotations are defined by $i\left(J_{k}\right)_{i j}=\epsilon_{i j k}$.
[1] M. Gomez-Bock, M. Mondragon, M. Muhlleitner, M. Spira, and P. M. Zerwas, arXiv:0712.2419.

[2] L. Randall, arXiv:0711.4360.

[3] M. Maniatis, A. von Manteuffel, O. Nachtmann, and F. Nagel, Eur. Phys. J. C 48, 805 (2006).

[4] P. M. Ferreira, R. Santos, and A. Barroso, Phys. Lett. B 603, 219 (2004); 629, 114(E) (2005); 632, 684 (2006).

[5] S. Davidson and H. E. Haber, Phys. Rev. D 72, 035004(E) (2005); 72, 099902 (2005).

[6] H.E. Haber and D. O'Neil, Phys. Rev. D 74, 015018 (2006); 74, 059905(E) (2006).

[7] J.F. Gunion and H.E. Haber, Phys. Rev. D 72, 095002 (2005).

[8] I. P. Ivanov, arXiv:0710.3490.

[9] I. P. Ivanov, Phys. Rev. D 75, 035001 (2007); 76, 039902(E) (2007).

[10] I. F. Ginzburg and M. Krawczyk, Phys. Rev. D 72, 115013 (2005).

[11] M. S. Carena and H. E. Haber, Prog. Part. Nucl. Phys. 50, 63 (2003).

[12] T. D. Lee, Phys. Rev. D 8, 1226 (1973); Phys. Rep. 9, 143 (1974).

[13] S. Weinberg, Phys. Rev. Lett. 37, 657 (1976).

[14] M. Sher, Phys. Rep. 179, 273 (1989).

[15] A. Barroso, P. M. Ferreira, R. Santos, and J. P. Silva, Phys. Rev. D 74, 085016 (2006).

[16] The terminology "horizontal transformations" and "basis transformations", as well as "horizontal group" and "basis transformation group", will be used interchangeably.
[17] W. Grimus and M. N. Rebelo, Phys. Rep. 281, 239 (1997).

[18] F. J. Botella and J. P. Silva, Phys. Rev. D 51, 3870 (1995); L. Lavoura and J. P. Silva, Phys. Rev. D 50, 4619 (1994).

[19] C. C. Nishi, Phys. Rev. D 74, 036003 (2006); 76, 119901(E) (2007).

[20] C. Jarlskog, Phys. Rev. Lett. 55, 1039 (1985). Also, Phys. Rev. D 35, 1685 (1987).

[21] G. C. Branco, M. N. Rebelo, and J. I. Silva-Marcos, Phys. Lett. B 614, 187 (2005).

[22] I. P. Ivanov, Phys. Lett. B 632, 360 (2006).

[23] A set of continuous minima (of different orbits) may exist in certain symmetric potentials [8].

[24] A. Barroso, P. M. Ferreira, and R. Santos, Phys. Lett. B 652, 181 (2007).

[25] C. C. Nishi, Phys. Rev. D 76, 055013 (2007).

[26] The mass squared of the physical charged scalar $h^{+}, m^{2}$, can be regarded as a scalar under $S U(2)_{H}$ by defining $m^{2}=\operatorname{Tr}[\langle\mathbb{M}\rangle]$.

[27] L. Lavoura, Phys. Rev. D 50, 7089 (1994).

[28] G. Barenboim, E. Lunghi, P. Paradisi, W. Porod, and O. Vives, arXiv:0712.3559.

[29] M. Krawczyk and D. Sokolowska, arXiv:0711.4900.

[30] S. Mantry, M. Trott, and M. B. Wise, Phys. Rev. D 77, 013006 (2008).

[31] This remark is due to Igor Ivanov.

[32] With more than one physical charged Higgs boson, their masses will enter as physical parameters instead of only one $m^{2}$.

[33] C. C. Nishi, Am. J. Phys. 73, 1160 (2005). 